\title{
ASYMPTOTICS OF APPROXIMATION OF FUNCTIONS BY CONJUGATE POISSON INTEGRALS
}

\begin{abstract}
Among the actual problems of the theory of approximation of functions one should highlight a wide range of extremal problems, in particular, studying the approximation of functional classes by various linear methods of summation of the Fourier series. In this paper, we consider the wellknown Lipschitz class $\operatorname{Lip}_{1} \alpha$, i.e. the class of continuous $2 \pi$-periodic functions satisfying the Lipschitz condition of order $\alpha, 0<\alpha \leq 1$, and the conjugate Poisson integral acts as the approximating operator. One of the relevant tasks at present is the possibility of finding constants for asymptotic terms of the indicated degree of smallness (the so-called Kolmogorov-Nikol'skii constants) in asymptotic distributions of approximations by the conjugate Poisson integrals of functions from the Lipschitz class in the uniform metric. In this paper, complete asymptotic expansions are obtained for the exact upper bounds of deviations of the conjugate Poisson integrals from functions from the class $\operatorname{Lip}_{1} \alpha$. These expansions make it possible to write down the Kolmogorov-Nikol'skii constants of the arbitrary order of smallness.
\end{abstract}

Key words and phrases: Poisson integral, asymptotic expansion, conjugate function, KolmogorovNikol'skii problem.

\footnotetext{
${ }^{1}$ Lesya Ukrainka East European National University, 13 Voli avenue, 43025, Lutsk, Ukraine

2 Institute of Mathematics, National Academy of Sciences of Ukraine, 3 Tereschenkivska str., 01601, Kyiv, Ukraine

E-mail: k.inna.80@gmail.com (Kal'chuk I.V.), kharkevich.juriy@gmail.com (Kharkevych Yu.I.), kate.shvai@gmail. com (Pozharska K.V.)
}

\section{INTRODUCTION}

Let $C$ be the space of $2 \pi$-periodic continuous functions equipped with the norm $\|f\|_{C}=\max _{t}|f(t)|$.

Denote by $W^{r}$ any set of $2 \pi$-periodic functions with absolutely continuous derivatives up to order $(r-1)$ such that ess $\sup _{t}\left|f^{(r)}(t)\right| \leq 1$.

The set of functions that are conjugate to those from the class $W^{r}$ is denoted by $\bar{W}^{r}$. That is

$$
\begin{gathered}
\bar{W}^{r}=\left\{\bar{f}: \bar{f}(x)=-\frac{1}{2 \pi} \int_{-\pi}^{\pi} f(x+t) \cot \frac{t}{2} d t=-\frac{1}{2 \pi} \int_{0}^{\pi} \psi_{x}(t) \cot \frac{t}{2} d t\right. \\
\left.\psi_{x}(t)=f(x+t)-f(x-t), \quad f \in W^{r}\right\} .
\end{gathered}
$$

Any $f \in C$ is contained in the class $\operatorname{Lip}_{1} \alpha, 0<\alpha \leq 1$, if

$$
\forall t_{1}, t_{2} \in \mathbb{R} \quad\left|f\left(t_{1}\right)-f\left(t_{2}\right)\right| \leq\left|t_{1}-t_{2}\right|^{\alpha} .
$$

$\mathrm{y} \Delta \mathrm{K} 517.5$

2010 Mathematics Subject Classification: 42A05, 41A60. 
Let us consider a boundary value problem (in the unit circle) for the equation $\Delta u=0$, where $\Delta$ is the Laplace operator in polar coordinates. We can rewrite this equation as follows

$$
\frac{\partial^{2} u}{\partial \rho^{2}}+\frac{1}{\rho} \frac{\partial u}{\partial \rho}+\frac{1}{\rho^{2}} \frac{\partial^{2} u}{\partial x^{2}}=0, \quad 0 \leq \rho<1, \quad-\pi \leq x \leq \pi .
$$

A solution $P_{\rho}(f ; x)$ of $(1)$ that satisfies the boundary conditions

$$
\left.u(\rho, x)\right|_{\rho=1}=f(x), \quad-\pi \leq x \leq \pi,
$$

where $f$ is a summable $2 \pi$-periodic function, is of the form

$$
P_{\rho}(f ; x)=\frac{1}{\pi} \int_{-\pi}^{\pi} f(x+t) K_{\rho}(t) d t
$$

where

$$
K_{\rho}(t)=\frac{1}{2}+\sum_{k=1}^{\infty} \rho^{k} \cos k t=\frac{1-\rho^{2}}{2\left(1-2 \rho \cos t+\rho^{2}\right)} .
$$

The quantity $P_{\rho}(f ; x)$ is called the Poisson integral of a function $f$, and, respectively, $K_{\rho}(t)$ is called the kernel of the Poisson integral.

In the paper, we consider the conjugate Poisson integral, i.e. the quantity of the following form

$$
\bar{P}_{\rho}(f ; x)=P_{\rho}(\bar{f} ; x)=-\frac{1}{\pi} \int_{-\pi}^{\pi} f(x+t) \bar{K}_{\rho}(t) d t
$$

where

$$
\bar{K}_{\rho}(t)=\sum_{k=1}^{\infty} \rho^{k} \sin k t=\frac{\rho \sin t}{1-2 \rho \cos t+\rho^{2}}
$$

is the kernel of the conjugate Poisson integral.

Let $\mathfrak{N} \subseteq C$ be a certain class of functions. According to Stepanets [12], the problem of establishment of asymptotic equalities for the quantity

$$
\mathcal{E}\left(\mathfrak{N} ; P_{\rho}\right)_{C}=\sup _{f \in \mathfrak{N}}\left\|f(\cdot)-P_{\rho}(f ; \cdot)\right\|_{C}
$$

is called the Kolmogorov-Nikol'skii problem.

If we determine the explicit form of a function $\varphi(\rho)$ such that

$$
\mathcal{E}\left(\mathfrak{N} ; P_{\rho}\right)_{C}=\varphi(\rho)+o(\varphi(\rho)) \quad \text { as } \rho \rightarrow 1-,
$$

then we say that the Kolmogorov-Nikol'skii problem for the Poisson integral $P_{\rho}$ is solved on the class $\mathfrak{N}$ in the metric of the space $C$.

Definition 1. A formal series $\sum_{n=0}^{\infty} g_{n}(\rho)$ is called a complete asymptotic expansion of a function $f(\rho)$ as $\rho \rightarrow 1-$, if for an arbitrary natural number $m$ the following equation holds

$$
f(\rho)=\sum_{n=0}^{m} g_{n}(\rho)+o\left(g_{m}(\rho)\right) \quad \text { as } \rho \rightarrow 1-,
$$

and $\forall n \in N$

$$
\left|g_{n+1}(\rho)\right|=o\left(\left|g_{n}(\rho)\right|\right) \quad \text { as } \rho \rightarrow 1-.
$$

In what follows, this fact we denote by

$$
f(\rho) \cong \sum_{n=0}^{\infty} g_{n}(\rho)
$$


Approximation properties of the method of approximation by Poisson integrals on classes of differentiable functions are well studied. The Kolmogorov-Nikol'skii problem for the Poisson integral on the classes $W^{1}$ was solved by Natanson in [10].

Timan [14] obtained the exact values of approximative characteristics $\mathcal{E}\left(W^{r} ; P_{\rho}\right)_{C}$. In the paper [9] Malei determined the complete asymptotic expansion of the upper bounds of deviations of Poisson integrals from functions of the class $W^{1}$. Later, this expansion was reproved by Stark [11].

The complete asymptotic expansion of the quantity $\mathcal{E}\left(W^{r} ; P_{\delta}\right)_{C}$ in powers of $\frac{1}{\delta}$ as $\delta \rightarrow \infty$ was obtained by Baskakov [2] in the case of $r=1,2,3$ and by Kharkevych, Kal'chuk [5] for any natural $r$. Later, the Kolmogorov-Nikol'skii problem for the Poisson integral on classes of differentiable functions was solved in works [7, 8, 15, 18-21]. Simultaneously, approximation properties of the method of approximation by Poisson integrals on classes of conjugate functions are studied not enough.

Note that the first estimates of $\mathcal{E}\left(\bar{W}^{1} ; P_{\rho}\right)_{C}$ were obtained by Nagy [13]. Later, the general expressions that allow one to get asymptotic expansions of the quantity $\mathcal{E}\left(\bar{W}^{r} ; P_{\delta}\right)_{C}$ in powers of $\frac{1}{\delta}$ as $\delta \rightarrow \infty$ were determined by Baskakov [1].

The present paper is an extension of the paper [6], where the corresponding results for the classes $\operatorname{Lip}_{1} 1$ were obtained in terms of $(1-\rho)$. In what follows, we establish a complete asymptotic expansion of the quantity

$$
\mathcal{E}\left(\operatorname{Lip}_{1} \alpha ; \bar{P}_{\rho}\right)_{C}=\sup _{f \in \operatorname{Lip}_{1} \alpha}\left\|\bar{f}(\cdot)-\bar{P}_{\rho}(f ; \cdot)\right\|_{C^{\prime}} \quad 0<\alpha \leq 1 .
$$

This expansion allows one to write down the Kolmogorov-Nikol'skii constants of an arbitrary order.

\section{MAin RESUlts}

The following statement is true.

Theorem 1. For $0<\alpha \leq 1$ the following complete asymptotic expansion holds as $\rho \rightarrow 1-$

$$
\begin{aligned}
\mathcal{E}\left(\operatorname{Lip}_{1} \alpha ; \bar{P}_{\rho}\right)_{C} & =\frac{2^{\alpha-1}}{\sin \frac{\alpha \pi}{2}}\left(\ln \frac{1}{\rho}\right)^{\alpha}-\frac{2^{\alpha}}{\pi} \sum_{k=0}^{\infty}(-1)^{k} \frac{\left(\frac{2}{\pi}\right)^{2(k+1)-\alpha}}{2 k-\alpha+2}\left(\ln \frac{1}{\rho}\right)^{2 k+2} \\
& +\frac{2^{\alpha}}{\pi \alpha} \sum_{k=0}^{\infty}(-1)^{k}\left(\ln \frac{1}{\rho}\right)^{2 k+2} \sum_{i=1}^{\infty} \int_{0}^{\left(\frac{\pi}{2}\right)^{\alpha}}\left(\left((2 i-1) \pi-u^{\frac{1}{\alpha}}\right)^{-2 k-3}\right. \\
& \left.-\left((2 i-1) \pi+u^{\frac{1}{\alpha}}\right)^{-2 k-3}+\left(2 \pi i+u^{\frac{1}{\alpha}}\right)^{-2 k-3}-\left(2 \pi i-u^{\frac{1}{\alpha}}\right)^{-2 k-3}\right) u^{\frac{1}{\alpha}} \mathrm{d} u .
\end{aligned}
$$

Proof. Note first, that the kernel of the conjugate Poisson integral (3) can be rewritten as

$$
\bar{K}_{\rho}(t)=\frac{1}{2} \cot \frac{t}{2}-\frac{1}{2} \cot \frac{t}{2} \cdot \frac{(1-\rho)^{2}}{1-2 \rho \cos t+\rho^{2}} .
$$

Whence, in view of $2 \pi$-periodicity of functions $f$, we get

$$
\bar{P}_{\rho}(f, x)-\bar{f}(x)=\frac{1}{2 \pi} \int_{-\pi}^{\pi} f(x+t) \cot \frac{t}{2} \cdot \frac{(1-\rho)^{2}}{1-2 \rho \cos t+\rho^{2}} d t
$$




$$
\begin{aligned}
& =\frac{1}{2 \pi} \int_{0}^{\pi}(f(x+t)-f(x-t)) \cot \frac{t}{2} \cdot \frac{(1-\rho)^{2}}{1-2 \rho \cos t+\rho^{2}} d t \\
& =\frac{1}{2 \pi} \int_{0}^{\frac{\pi}{2}}(f(x+t)-f(x-t)) \cot \frac{t}{2} \cdot \frac{(1-\rho)^{2}}{1-2 \rho \cos t+\rho^{2}} d t \\
& \quad-\frac{1}{2 \pi} \int_{\frac{\pi}{2}}^{\pi}(f(2 \pi+x-t)-f(x+t)) \cot \frac{t}{2} \cdot \frac{(1-\rho)^{2}}{1-2 \rho \cos t+\rho^{2}} d t .
\end{aligned}
$$

For $0 \leq t \leq \pi$

$$
\cot \frac{t}{2} \cdot \frac{(1-\rho)^{2}}{1-2 \rho \cos t+\rho^{2}} \geq 0,
$$

functions $f$ belong the class $\operatorname{Lip}_{1} \alpha$, therefore it holds

$$
\begin{aligned}
\left|\bar{P}_{\rho}(f, x)-\bar{f}(x)\right| \leq & \frac{2^{\alpha}}{2 \pi} \int_{0}^{\frac{\pi}{2}} t^{\alpha} \cot \frac{t}{2} \cdot \frac{(1-\rho)^{2}}{1-2 \rho \cos t+\rho^{2}} d t \\
& +\frac{2^{\alpha}}{2 \pi} \int_{\frac{\pi}{2}}^{\pi}(\pi-t)^{\alpha} \cot \frac{t}{2} \cdot \frac{(1-\rho)^{2}}{1-2 \rho \cos t+\rho^{2}} d t .
\end{aligned}
$$

Let $[g(t)]_{2 \pi}$ be an odd $2 \pi$-periodic extension of the function $g$ of the form

$$
g(t)=g(\alpha, t)= \begin{cases}t^{\alpha}, & 0 \leq t \leq \frac{\pi}{2} \\ (\pi-t)^{\alpha}, & \frac{\pi}{2} \leq t \leq \pi .\end{cases}
$$

The function $f^{*}(t):=2^{\alpha-1}[g(t)]_{2 \pi}$ belongs to the class $\operatorname{Lip}_{1} \alpha$, and we can see that the right hand side of (5) coincides with $\bar{P}_{\rho}\left(f^{*}, 0\right)-\bar{f}^{*}(0)$. Indeed, taking into account that $f^{*}$ is odd, we get

$$
\begin{aligned}
\bar{P}_{\rho}\left(f^{*}, 0\right)-\bar{f}^{*}(0) & =\frac{1}{2 \pi} \int_{-\pi}^{\pi} f^{*}(t) \cot \frac{t}{2} \cdot \frac{(1-\rho)^{2}}{1-2 \rho \cos t+\rho^{2}} d t \\
& =\frac{1}{\pi} \int_{0}^{\pi} f^{*}(t) \cot \frac{t}{2} \cdot \frac{(1-\rho)^{2}}{1-2 \rho \cos t+\rho^{2}} d t .
\end{aligned}
$$

The right hand side of (7) coincides with the right hand side of (5). Hence

$$
\mathcal{E}\left(\operatorname{Lip}_{1} \alpha ; \bar{P}_{\rho}\right)_{C}=\left|\bar{P}_{\rho}\left(f^{*}, 0\right)-\bar{f}^{*}(0)\right| .
$$

Let us rewrite the kernel of the conjugate Poisson integral (3) in the following form

$$
\bar{K}_{\rho}(t)=\sum_{k=1}^{\infty} e^{\ln \rho^{k}} \sin k t=\sum_{k=1}^{\infty} e^{-\ln \left(\frac{1}{\rho}\right) k} \sin k t .
$$

It is known, that the Fourier cosine transform of the function $e^{-\beta t}$ takes the form [3, Ch. VII],

$$
\Phi_{c}(u)=\frac{1}{\sqrt{2 \pi}}\left\{\frac{t+u}{\beta^{2}+(t+u)^{2}}+\frac{t-u}{\beta^{2}+(t-u)^{2}}\right\} .
$$


Further we shall need the Poisson formula [16, Ch. II]:

$$
\sqrt{\gamma}\left(\frac{\Phi_{c}(0)}{2}+\sum_{n=1}^{\infty} \Phi_{c}(n \gamma)\right)=\sqrt{\omega}\left(\frac{f(0)}{2}+\sum_{n=1}^{\infty} f(n \omega)\right),
$$

where $\omega \gamma=2 \pi, \omega>0$. Setting $\omega=1, \gamma=2 \pi$ in (11) and taking into account (10) with $\beta=\ln \frac{1}{\rho}$, from (9) we obtain

$$
\begin{aligned}
\bar{K}_{\rho}(t) & =\left.\frac{1}{2}\left(e^{-\ln \left(\frac{1}{\rho}\right) k} \sin k t\right)\right|_{k=0}+\sum_{k=1}^{\infty}\left(e^{-\ln \left(\frac{1}{\rho}\right) k} \sin k t\right)=\sqrt{2 \pi}\left\{\frac{1}{2} \Phi_{c}(0)+\sum_{k=1}^{\infty} \Phi_{c}(2 \pi k)\right\} \\
& =\frac{t}{\beta^{2}+t^{2}}+\sum_{k=1}^{\infty}\left(\frac{t+2 \pi k}{\beta^{2}+(t+2 \pi k)^{2}}+\frac{t-2 \pi k}{\beta^{2}+(t-2 \pi k)^{2}}\right) .
\end{aligned}
$$

Therefore, combining (2) and (12), we can write the conjugate Poisson integral in the following equivalent form

$$
\begin{aligned}
\bar{P}_{\rho}\left(f^{*}, 0\right)=-\frac{1}{\pi} \int_{-\pi}^{\pi} f^{*}(t) \frac{t}{\beta^{2}+t^{2}} d t & -\frac{1}{\pi} \int_{-\pi}^{\pi} f^{*}(t) \sum_{k=1}^{\infty} \frac{t+2 \pi k}{\beta^{2}+(t+2 \pi k)^{2}} d t \\
& -\frac{1}{\pi} \int_{-\pi}^{\pi} f^{*}(t) \sum_{k=1}^{\infty} \frac{t-2 \pi k}{\beta^{2}+(t-2 \pi k)^{2}} d t=I_{1}-I_{2}-I_{3} .
\end{aligned}
$$

Now we proceed to calculating of the term $I_{2}$ :

$$
\begin{aligned}
I_{2}= & \frac{1}{\pi} \int_{-\pi}^{\pi} f^{*}(t) \sum_{k=1}^{\infty} \frac{t+2 \pi k}{\beta^{2}+(t+2 \pi k)^{2}} d t=\frac{1}{\pi} \int_{-\pi}^{\pi} f^{*}(t) \frac{t+2 \pi}{\beta^{2}+(t+2 \pi)^{2}} d t \\
& +\frac{1}{\pi} \int_{-\pi}^{\pi} f^{*}(t) \frac{t+4 \pi}{\beta^{2}+(t+4 \pi)^{2}} d t+\cdots=I_{2,1}+I_{2,2}+\cdots .
\end{aligned}
$$

Making appropriate substitutions in $I_{2,1}, I_{2,2}, \cdots$, we get

$$
I_{2,1}=\frac{1}{\pi} \int_{\pi}^{3 \pi} f^{*}(t) \frac{t}{\beta^{2}+t^{2}} d t, \quad I_{2,2}=\frac{1}{\pi} \int_{3 \pi}^{5 \pi} f^{*}(t) \frac{t}{\beta^{2}+t^{2}} d t, \cdots .
$$

Hence, from (14), we obtain

$$
I_{2}=\frac{1}{\pi} \int_{\pi}^{+\infty} f^{*}(t) \frac{t}{\beta^{2}+t^{2}} d t
$$

One can verify that the term $I_{3}$ takes the following form

$$
I_{3}=\frac{1}{\pi} \int_{-\pi}^{\pi} f^{*}(t) \sum_{k=1}^{\infty} \frac{t-2 \pi k}{\beta^{2}+(t-2 \pi k)^{2}} d t=\frac{1}{\pi} \int_{-\infty}^{-\pi} f^{*}(t) \frac{t}{\beta^{2}+t^{2}} d t
$$

Combining (13) with (15) and (16), we obtain

$$
\bar{P}_{\rho}\left(f^{*}, 0\right)=-\frac{1}{\pi} \int_{-\infty}^{+\infty} f^{*}(t) \frac{t}{\beta^{2}+t^{2}} d t .
$$


It is known [12, p. 93], that a conjugate for $f$ function can be represented in the form

$$
\bar{f}(x)=-\frac{1}{\pi} \int_{-\infty}^{+\infty} \frac{f(x+t)}{t} d t
$$

Therefore, from (17) and (18), taking into account that the function $f^{*}$ is odd, we get

$$
\begin{aligned}
\bar{P}_{\rho}\left(f^{*}, 0\right)-\bar{f}^{*}(0)=\frac{\beta^{2}}{\pi} \int_{-\infty}^{+\infty} \frac{f^{*}(t)}{t\left(\beta^{2}+t^{2}\right)} d t & =\frac{\beta^{2}}{\pi}\left(\int_{-\infty}^{0}+\int_{0}^{+\infty}\right) \frac{f^{*}(t)}{t\left(\beta^{2}+t^{2}\right)} d t \\
& =\frac{2 \beta^{2}}{\pi} \int_{0}^{+\infty} f^{*}(t) \frac{1}{t\left(\beta^{2}+t^{2}\right)} d t .
\end{aligned}
$$

Hence, from (8) and (19), we have

$$
\mathcal{E}\left(\operatorname{Lip}_{1} \alpha ; \bar{P}_{\rho}\right)_{C}=\frac{2^{\alpha} \beta^{2}}{\pi} \int_{0}^{+\infty} \frac{[g(t)]_{2 \pi}}{t\left(\beta^{2}+t^{2}\right)} d t=\frac{2^{\alpha} \beta^{2}}{\pi}\left(\int_{0}^{\frac{\pi}{2}}+\int_{\frac{\pi}{2}}^{+\infty}\right) \frac{[g(t)]_{2 \pi}}{t\left(\beta^{2}+t^{2}\right)} d t .
$$

From (6) we get

$$
\begin{aligned}
\int_{0}^{\frac{\pi}{2}} \frac{[g(t)]_{2 \pi}}{t\left(\beta^{2}+t^{2}\right)} d t=\int_{0}^{\frac{\pi}{2}} \frac{t^{\alpha}}{t\left(t^{2}+\beta^{2}\right)} d t & =\int_{0}^{\frac{\pi}{2}} \frac{\left(\frac{t}{\beta}\right)^{\alpha-1}}{\left(\frac{t}{\beta}\right)^{2}+1} \beta^{\alpha-2} \mathrm{~d}\left(\frac{t}{\beta}\right) \\
& =\beta^{\alpha-2}\left(\int_{0}^{+\infty}-\int_{\frac{\pi}{2 \beta}}^{+\infty}\right) \frac{u^{\alpha-1}}{1+u^{2}} \mathrm{~d} u .
\end{aligned}
$$

According to [4, p. 306]

$$
\int_{0}^{\infty} \frac{u^{\alpha-1}}{1+u^{2}} \mathrm{~d} u=\frac{\pi}{2} \operatorname{cosec} \frac{\pi \alpha}{2} .
$$

Let us make transformations in the second integral from the right-hand side of (21), applying geometric series

$$
\begin{aligned}
\int_{\frac{\pi}{2 \beta}}^{\infty} \frac{u^{\alpha-1}}{1+u^{2}} \mathrm{~d} u=\int_{\frac{\pi}{2 \beta}}^{\infty} \frac{1}{u^{3-\alpha}} \cdot \frac{1}{1-\left(-\frac{1}{u^{2}}\right)} \mathrm{d} u & =\int_{\frac{\pi}{2 \beta}}^{\infty} \sum_{k=0}^{\infty}(-1)^{k} \frac{1}{u^{2 k+3-\alpha}} \mathrm{d} u \\
& =\sum_{k=0}^{\infty}(-1)^{k} \frac{\left(\frac{2 \beta}{\pi}\right)^{2 k+2-\alpha}}{2 k+2-\alpha}
\end{aligned}
$$

Combining formulas (21), (22) and (23), we obtain

$$
\int_{0}^{\frac{\pi}{2}} \frac{[g(t)]_{2 \pi}}{t\left(\beta^{2}+t^{2}\right)} d t=\left(\ln \frac{1}{\rho}\right)^{\alpha-2}\left(\frac{\pi}{2 \sin \frac{\pi \alpha}{2}}-\sum_{k=0}^{\infty} \frac{(-1)^{k}}{2 k+2-\alpha}\left(\frac{2 \ln \frac{1}{\rho}}{\pi}\right)^{2 k+2-\alpha}\right) .
$$


Then, we use geometric series for calculating the second integral from the right-hand side of (20):

$$
\int_{\frac{\pi}{2}}^{+\infty} \frac{[g(t)]_{2 \pi}}{t\left(\beta^{2}+t^{2}\right)} d t=\int_{\frac{\pi}{2}}^{+\infty} \frac{[g(t)]_{2 \pi}}{t^{3}\left(1+\frac{\beta^{2}}{t^{2}}\right)} d t=\sum_{k=0}^{\infty}(-1)^{k}\left(\ln \frac{1}{\rho}\right)^{2 k} \int_{\frac{\pi}{2}}^{+\infty} \frac{[g(t)]_{2 \pi}}{t^{3+2 k}} d t .
$$

From (24) and (25), we get

$$
\begin{aligned}
\mathcal{E}\left(\operatorname{Lip}_{1} \alpha ; \bar{P}_{\rho}\right)_{C} & =\left(2 \ln \frac{1}{\rho}\right)^{\alpha} \frac{1}{2 \sin \frac{\alpha \pi}{2}} \\
& +\frac{2^{\alpha}}{\pi} \sum_{k=0}^{\infty}(-1)^{k}\left(\ln \frac{1}{\rho}\right)^{2(k+1)}\left\{\int_{\frac{\pi}{2}}^{+\infty} \frac{[g(t)]_{2 \pi}}{t^{3+2 k}} d t-\frac{\left(\frac{2}{\pi}\right)^{2 k+2-\alpha}}{2 k+2-\alpha}\right\} d t .
\end{aligned}
$$

For the function $[g(t)]_{2 \pi}$ on $\left[\frac{\pi}{2} ;+\infty\right)$ the following relations hold

$$
[g(t)]_{2 \pi}= \begin{cases}(-t+(2 i-1) \pi)^{\alpha}, & t \in\left[\frac{\pi}{2}+4(i-1) \frac{\pi}{2} ; \frac{\pi}{2}+(4 i-3) \frac{\pi}{2}\right], \\ -(t-(2 i-1) \pi)^{\alpha}, & t \in\left[\frac{\pi}{2}+(4 i-3) \frac{\pi}{2} ; \frac{\pi}{2}+(4 i-2) \frac{\pi}{2}\right], \\ -(-t+2 \pi i)^{\alpha}, & t \in\left[\frac{\pi}{2}+(4 i-2) \frac{\pi}{2} ; \frac{\pi}{2}+(4 i-1) \frac{\pi}{2}\right], \\ (t-2 \pi i)^{\alpha}, & t \in\left[\frac{\pi}{2}+(4 i-1) \frac{\pi}{2} ; \frac{\pi}{2}+4 i \frac{\pi}{2}\right],\end{cases}
$$

where $i=1,2, \ldots$. Splitting the integral $\int_{\frac{\pi}{2}}^{+\infty} \frac{[g(t)]_{2 \pi}}{t^{3+2 k}} d t$ into the sum of integrals and making corresponding substitutions in each of them, we get (4).

The theorem is proved.

Results of the theorem give us an opportunity to write down the Kolmogorov-Nikil'skii constants of an arbitrary order in asymptotic expansions in terms of $\ln \frac{1}{\rho}$ as $\rho \rightarrow 1-$.

Let us consider the class of functions $\operatorname{Lip}_{1} 1$. The following statement holds.

Corollary 1. The complete asymptotic expansion holds as $\rho \rightarrow 1-$

$$
\begin{aligned}
\mathcal{E}\left(\operatorname{Lip}_{1} 1 ; \bar{P}_{\rho}\right)_{C} & =\ln \frac{1}{\rho}-\frac{1}{\pi}\left(\ln \frac{1}{\rho}\right)^{2}+\sum_{k=1}^{\infty} \frac{(-1)^{k}}{(2 k+1)(k+1)} \\
& \times\left(\sum_{i=1}^{\infty}\left(\frac{1}{(4 i-1)^{2 k+1}}-\frac{1}{(4 i+1)^{2 k+1}}\right)-1\right)\left(\frac{2}{\pi} \ln \frac{1}{\rho}\right)^{2 k+2} .
\end{aligned}
$$

Proof. Putting $\alpha=1$ in (20), we obtain

$$
\mathcal{E}\left(\operatorname{Lip}_{1} 1 ; \bar{P}_{\rho}\right)_{C}=\frac{2 \beta^{2}}{\pi} \int_{0}^{+\infty} \frac{\left[g_{1}(t)\right]_{2 \pi}}{t\left(\beta^{2}+t^{2}\right)} d t
$$

where $\left[g_{1}(t)\right]_{2 \pi}$ is as it was introduced earlier, an odd $2 \pi$-periodic extension of the function $g_{1}$ of the form

$$
g_{1}(t)= \begin{cases}t, & 0 \leq t \leq \frac{\pi}{2} \\ \pi-t, & \frac{\pi}{2} \leq t \leq \pi\end{cases}
$$


Making transformations that are analogous to that in (21)-(25), from (27) we get

$$
\begin{aligned}
\mathcal{E}\left(\operatorname{Lip}_{1} 1 ; \bar{P}_{\rho}\right)_{C} & =\ln \frac{1}{\rho}+\frac{2}{\pi} \sum_{k=0}^{\infty}(-1)^{k}\left(\ln \frac{1}{\rho}\right)^{2 k+2} \\
& \times\left(\int_{\frac{\pi}{2}}^{+\infty} \frac{\left[g_{1}(t)\right]_{2 \pi}}{t^{3+2 k}} d t-\frac{1}{2 k+1}\left(\frac{2}{\pi}\right)^{2 k+1}\right) .
\end{aligned}
$$

To calculate the integral in the right-hand side of (28), let us write it down as a sum of the integrals on corresponding intervals. For this reason we use the following form of the function $g_{1}$ :

$$
\left[g_{1}(t)\right]_{2 \pi}= \begin{cases}-t+(2 i-1) \pi, & t \in\left[-\frac{\pi}{2}+(2 i-1) \pi ;-\frac{\pi}{2}+2 \pi i\right], \\ t-2 \pi i, & t \in\left[-\frac{\pi}{2}+2 \pi i ;-\frac{\pi}{2}+(2 i+1) \pi\right] .\end{cases}
$$

We obtain

$$
\int_{\frac{\pi}{2}}^{+\infty} \frac{\left[g_{1}(t)\right]_{2 \pi}}{t^{3+2 k}} d t=\sum_{i=1}^{\infty}\left(\int_{-\frac{\pi}{2}+(2 i-1) \pi}^{-\frac{\pi}{2}+2 \pi i} \frac{-t+(2 i-1) \pi}{t^{3+2 k}} d t+\int_{-\frac{\pi}{2}+2 \pi i}^{-\frac{\pi}{2}+(2 i+1) \pi} \frac{t-2 \pi i}{t^{3+2 k}} d t\right) .
$$

Having calculated the integral on the right-hand side of (29) and made corresponding transformations, we obtain

$$
\begin{aligned}
\int_{\frac{\pi}{2}}^{+\infty} \frac{\left[g_{1}(t)\right]_{2 \pi}}{t^{3+2 k}} d t & =\left(\frac{2}{\pi}\right)^{2 k+1} \frac{1}{(2 k+1)(k+1)} \\
& \times\left(k+\sum_{i=1}^{\infty}\left(\frac{1}{(4 i-1)^{2 k+1}}-\frac{1}{(4 i+1)^{2 k+1}}\right)\right) .
\end{aligned}
$$

Taking into account (30), from (28) we get (26).

The Corollary 1 is proved.

In the paper we have also obtained another form of the expansion (26), in terms of the generalized Riemann zeta function (the Hurwitz zeta function) (see definition, e.g., [17, Ch. XIII]). It is quite relevant because in approximation of functions by the Poisson integrals we obtain asymptotic expansions with non-explicit form of the coefficients. The Hurwitz zeta function gives a possibility to get sharp values of the Kolmogorov-Nikil'skii constants.

Corollary 2. The complete asymptotic expansion holds as $\rho \rightarrow 1-$

$$
\begin{aligned}
\mathcal{E}\left(\operatorname{Lip}_{1} 1 ; \bar{P}_{\rho}\right)_{C} & =\ln \frac{1}{\rho}-\frac{1}{\pi}\left(\ln \frac{1}{\rho}\right)^{2}+\sum_{k=1}^{\infty} \frac{(-1)^{k}}{(2 k+1)(k+1)} \\
& \times\left(\frac{1}{4^{2 k+1}}\left\{\zeta\left(2 k+1 ; \frac{3}{4}\right)-\zeta\left(2 k+1 ; \frac{5}{4}\right)\right\}-1\right)\left(\frac{2}{\pi} \ln \frac{1}{\rho}\right)^{2 k+2},
\end{aligned}
$$

where $\zeta(z ; q)=\sum_{n=0}^{\infty} \frac{1}{(q+n)^{2}}, \operatorname{Re} z>1$, is the Hurwitz zeta function. 
Proof. Taking into account that

$$
\begin{aligned}
& \sum_{i=1}^{\infty} \frac{1}{(4 i-1)^{2 k+1}}=\frac{1}{4^{2 k+1}} \sum_{i=0}^{\infty} \frac{1}{\left(i+\frac{3}{4}\right)^{2 k+1}}=\frac{1}{4^{2 k+1}} \zeta\left(2 k+1 ; \frac{3}{4}\right), \\
& \sum_{i=1}^{\infty} \frac{1}{(4 i+1)^{2 k+1}}=\frac{1}{4^{2 k+1}} \zeta\left(2 k+1 ; \frac{5}{4}\right),
\end{aligned}
$$

from (30) we derive

$$
\begin{aligned}
\int_{\frac{\pi}{2}}^{+\infty} \frac{\left[g_{1}(t)\right]_{2 \pi}}{t^{3+2 k}} d t & =\left(\frac{2}{\pi}\right)^{2 k+1} \frac{1}{(2 k+1)(k+1)} \\
& \times\left(k+\frac{1}{4^{2 k+1}}\left(\zeta\left(2 k+1 ; \frac{3}{4}\right)-\zeta\left(2 k+1 ; \frac{5}{4}\right)\right)\right) .
\end{aligned}
$$

The Corollary 2 is proved.

Note that (32) holds for $k=1,2, \ldots$ In the case $k=0$ the Hurwitz zeta function is not determined. Therefore the corresponding coefficient is individually calculated in the expansion (31).

Note also that Corollary 1 is a generalization of the B. Nagy result [13]. The estimation

$$
\mathcal{E}\left(\operatorname{Lip}_{1} 1 ; \bar{P}_{\rho}\right)_{C}=\ln \frac{1}{\rho}+O\left(\left(\ln \frac{1}{\rho}\right)^{2}\right), \quad \rho \rightarrow 1-
$$

follows from Corollary 1 that coincides with the indicated result.

\section{REFERENCES}

[1] Baskakov V.A. Asymptotic estimates for approximation of conjugate functions by conjugate Abel-Poisson integrals. Appl. Funct. Analysis to Approx. Theory 1975, 5, 14-20. (in Russian)

[2] Baskakov V.A. Some properties of operators of Abel-Poisson type. Math. Notes 1975, 17 (2), 101-107. doi: 10.1007/BF01161864

[3] Ditkin V.A., Prudnikov A.P. Integral transforms and operational calculus. Pergamon Press, Oxford, New York, 1965.

[4] Gradshtein I.S., Ryzhik I.M. Tables of Integrals, Sums, Series, and Products. Fizmatgiz, Moscow, 1963. (in Russian)

[5] Kharkevych Yu.I., Kal'chuk I.V. Complete asymptotics of the approximation of function from the Sobolev classes by the Poisson integrals. Acta Comment. Univ. Tartu. Math. 2018, 22 (1), 23-36. doi:10.12697/ACUTM.2018.22.03

[6] Kharkevych Yu.I., Pozharska K.V. Asymptotics of approximation of conjugate functions by Poisson Integrals. Acta Comment. Univ. Tartu. Math. 2018, 22 (2), 235-243. doi:10.12697/ ACUTM.2018.22.19

[7] Kharkevich Yu.I., Stepanyuk T.A. Approximation properties of Poisson integrals for the classes $C_{\beta}^{\psi} H^{\alpha}$. Math. Notes 2014, 96 (6), 1008-1019. doi:10.1134/S0001434614110406

[8] Kharkevych Yu.I., Zhyhallo T.V. Approximation of $(\psi, \beta)$-differentiable functions defined on the real axis by AbelPoisson operators. Ukrainian Math. J. 2005, 57 (8), 1297-1315. doi:10.1007/s11253-005-0262-z

[9] Malei L.V. Sharp estimate for approximation of quasismooth functions by Poisson integrals. Dokl. Akad. Nauk SSSR Ser. Fiz.-Tekh. 1961, 3, 25-32. (in Russian) 
[10] Natanson I.P. On the order of approximation of a continuous $2 \pi$-periodic function by its Poisson integral. Dokl. Akad. Nauk SSSR 1950, 72 (1), 11-14. (in Russian)

[11] Stark E.L. The complete asymptotic expansion for the measure of approximation of Abel-Poisson's singular integral for Lip 1. Math. Notes 1973, 13 (1), 14-18. doi:10.1007/BF01093622

[12] Stepanets A.I. Uniform approximation by trigonometric polynomials. Naukova Dumka, Kiev, 1981. (in Russian)

[13] Sz.-Nagy B. Sur l'ordre de l'approximation d'une fonction par son intégrale de Poisson. Acta Math. Acad. Sci. Hungar 1950, 1, 183-188.

[14] Timan A.F. Sharp estimate for a remainder in the approximation of periodic differentiable functions by Poisson integrals. Dokl. Akad. Nauk SSSR 1950, 74 (1), 17-20. (in Russian)

[15] Timan M.F. The Approximation and the properties of periodic functions. Naukova Dumka, Kiev, 2009. (in Russian)

[16] Titchmarsh E.C. Introduction to the theory of Fourier integrals. Clarendon Press, Oxford, 1948.

[17] Whittaker E.T., Watson G.N. A course of modern analysis. Cambridge University Press, 1927.

[18] Zhyhallo K.M., Kharkevych Yu.I. Complete asymptotics of the deviation of a class of differentiable functions from the set of their harmonic Poisson integrals. Ukrainian Math. J. 2002, 54 (1), 51-63. doi:10.1023/ A:1019789402502

[19] Zhyhallo K.M., Kharkevych Yu.I. Approximation of conjugate differentiable functions by their Abel-Poisson integrals. Ukrainian Math. J. 2009, 61 (1), 86-98. doi:10.1007/s11253-009-0196-y

[20] Zhyhallo T.V., Kharkevych Yu.I. Approximation of $(\psi, \beta)$-differentiable functions by Poisson integrals in the uniform metric. Ukrainian Math. J. 2009, 61 (11), 1757-1779. doi:10.1007/s11253-010-0311-0

[21] Zhyhallo T.V., Kharkevych Yu.I. Approximation of functions from the class $C_{\beta, \infty}^{\psi}$ by Poisson integrals in the uniform metric. Ukrainian Math. J. 2009, 61 (12), 1893-1914. doi:10.1007/s11253-010-0321-y

Received 02.01.2020

Кальчук І.В., Харкевич Ю.І., Пожарська К.В. Асимптотика наближення функиій спряженими інтегралами Пуассона // Карпатські матем. публ. - 2020. — Т.12, №1. — С. 138-147.

Актуальними задачами теорії наближення функцій $є$ розв'язання широкого кола екстремальних задач, зокрема, дослідження питань апроксимації функціональних класів різними лінійними методами підсумовування рядів Фур'є. В даній роботі розглядається відомий клас $\Lambda$ іпшиця $\operatorname{Lip}_{1} \alpha$, тобто клас неперервних $2 \pi$-періодичних функцій, що задовольняють умову Аіпшиця порядку $\alpha, 0<\alpha \leq 1$, а в якості наближаючого оператора виступає спряжений інтеграл Пуассона. Аосить актуальною задачею на даний час $є$ можливість знаходження констант при асимптотичних доданках вказаного степеня малості (так званих констант КолмогороваНікольського) в асимптотичних розкладах величин наближень спряженими інтегралами Пуассона функцій з класу Аіпшиця в рівномірній метриці. В роботі отримано повні асимптотичні розклади для точних верхніх меж відхилень спряжених інтегралів Пуассона від функцій $з$ класу $\operatorname{Lip}_{1} \alpha$. Аані розклади дають можливість записати константи Колмогорова-Нікольського довільного порядку малості.

Ключові слова і фрази: інтеграл Пуассона, асимптотичний розклад, спряжена функція, задача Колмогорова-Нікольського. 